\title{
Fragile topology in line-graph lattices with two, three, or four gapped flat bands
}

\author{
Christie S. Chiu $\odot$ \\ Department of Electrical Engineering, Princeton University, Princeton, New Jersey 08540, USA \\ and Princeton Center for Complex Materials, Princeton University, Princeton, New Jersey 08540, USA \\ Da-Shuai Ma \\ Key Laboratory of Advanced Optoelectronic Quantum Architecture and Measurement (MOE), Beijing Key Laboratory of Nanophotonics \\ and Ultrafine Optoelectronic Systems, and School of Physics, Beijing Institute of Technology, Beijing 100081, China \\ and Department of Physics, Princeton University, Princeton, New Jersey 08540, USA \\ Zhi-Da Song and B. Andrei Bernevig \\ Department of Physics, Princeton University, Princeton, New Jersey 08540, USA \\ Andrew A. Houck ${ }^{*}$ \\ Department of Electrical Engineering, Princeton University, Princeton, New Jersey 08540, USA
}

(Received 26 October 2020; accepted 8 December 2020; published 23 December 2020)

\begin{abstract}
The geometric properties of a lattice can have profound consequences on its band spectrum. For example, symmetry constraints and geometric frustration can give rise to topologicially nontrivial and dispersionless bands, respectively. Line-graph lattices are a perfect example of both of these features: Their lowest energy bands are perfectly flat, and here we develop a formalism to connect some of their geometric properties with the presence or absence of fragile topology in their flat bands. This theoretical work will enable experimental studies of fragile topology in several types of line-graph lattices, most naturally suited to superconducting circuits.
\end{abstract}

DOI: 10.1103/PhysRevResearch.2.043414

\section{INTRODUCTION}

Fragile topology is a property of a set of "Wannierobstructed" gapped electronic bands whose Wannier obstruction can be resolved by adding select trivial bands [1-13]. This Wannier obstruction refers to the inability to describe all states in these bands by exponentially localized symmetric Wannier functions, known as the atomic limit. "Extended" states are then required, much like the edge states of topological insulators [14-22]; crucially, however, the stable topology of these materials differs from fragile topology because it is robust to the addition of trivial bands. Additionally, the extended states of fragile phases generally do not exist at the edge. Recent theoretical and experimental work has found that fragile phases violate the bulk-boundary correspondence, but instead exhibit gapless edges under "twisted" boundary conditions $[23,24]$. Moreover, the fragile topology of electronic states also manifests itself in the contribution to the superfluid weight in the superconducting phase [25-28] and the level crossings in Hofstadter spectrum under magnetic field $[29,30]$.

\footnotetext{
*aahouck@princeton.edu

Published by the American Physical Society under the terms of the Creative Commons Attribution 4.0 International license. Further distribution of this work must maintain attribution to the author(s) and the published article's title, journal citation, and DOI.
}

Fragile topology can also be characterized under the theory of topological quantum chemistry, which classifies topological bands by classifying all possible atomic limits based on crystallographic symmetries $[1,31,32]$. Under this theory, atomic limits are described by elementary band representations (EBRs) [33-37]; while atomic bands can be written purely as a sum of EBRs, fragile topological bands cannot $[2,3]$. Instead, they can be written as sums and differences of EBRs, such that the inclusion of trivial bands can render the entire set of bands trivial. In this work, we mainly focus on the so-called eigenvalue fragile states whose irreducible representations (irreps) in momentum space cannot be written as sums of EBRs.

Less recently, theoretical work has also predicted that nearly flat bands with stable topology may give rise to fractional quantum Hall states at high temperatures or zero magnetic field [38-41]. However, to our knowledge no exact flat bands with stable topology have been found in lattice models. On the other hand, fragile topological bands can be exactly flat, for example, in magic-angle twisted bilayer graphene [42-47]. For exact flatness, then, fragile topological bands provide an ideal platform for studies of strongly interacting quantum phases [48]. Recent works [45,49-52] have shown that the partially filled fragile-topological flat bands in twisted bilayer graphene could form various correlated insulating phases, including the Chern insulator phase, under different parameters. It has also been shown that, remarkably, the Chern insulator phase originates from the fragile topol- 
ogy, which allows a natural choice of the Chern band basis [49,53-55].

Entire classes of lattices are known to have exactly flat bands, for example, bipartite lattices with an unequal number of vertices in each part [56] or certain types of "line-graph lattices" [57]. However, apart from directly computing the representation of specific flat-band systems, it is not generally known whether these bands are topological and, if so, whether the topology is stable or fragile.

Here we consider line-graph lattices of "regular" lattices, defined by the attribute that every vertex has the same coordination number. The band spectra of these lattices have flat bands as their lowest energy bands. Although the topology of these bands can be computed via topological quantum chemistry, this must be done on a case-by-case basis. We develop a framework for analyzing the topology of line-graph-lattice flat bands for entire families of lattices, drawing connections between simple geometric attributes of the lattices and their flat-band representations. With this framework, we identify such families whose flat bands have fragile topology, as well as families of line-graph lattices whose flat bands are topologically trivial but that, after certain perturbations, can be split into fragile topological flat bands and topologically trivial dispersive bands. These results can inform experimental simulations of line-graph lattices for studies of fragile topology; in particular, these lattices are quite natural to simulate with coplanar waveguide resonators in quantum circuits because the line-shaped resonators act as lattice vertices for microwave photons, with tunneling between vertices made possible through capacitive coupling at the resonator ends [58].

A line graph $L(X)$ can be formed from any graph $X$ (which we will refer to as the root graph) by placing a vertex $v_{L(X), i}$ on each edge $e_{X, i}$ of $X$ and connecting vertices $v_{L(X), i}$ and $v_{L(X), j}$ if their corresponding edges $e_{X, i}$ and $e_{X, j}$ are adjacent, i.e., share a common vertex. We then define the tight-binding Hamiltonian

$$
\hat{H}=\sum_{\langle i, j\rangle} \hat{a}_{i}^{\dagger} \hat{a}_{j}+\hat{a}_{j}^{\dagger} \hat{a}_{i},
$$

where the sum is taken over all adjacent vertices $v_{L(x), i}$ and $v_{L(X), j}$, representing amplitude-1 hopping of spinless bosons $\hat{a}_{i}$ between adjacent vertices in the line graph.

There are several properties of line graphs, discussed further in Appendix A of the Supplemental Material with examples [59], that are relevant to this work:

LG1 If $X$ is a periodic lattice, $L(X)$ is as well.

LG2 Any symmetries of $X$ are inherited by $L(X)$; i.e., the space group of $X$ is the same as that of $L(X)$.

LG3 As a consequence of the line-graph construction, every vertex $v_{X, i}$ of the root graph gives rise to a "complete subgraph" in the line graph, where a complete subgraph is defined as a subset of $k$ vertices and binomial coefficient $\left(\begin{array}{l}k \\ 2\end{array}\right)$ edges for which all pairs of vertices are connected by one of the edges (i.e., "fully connected"). In these complete subgraphs, $k$ will be equal to the coordination number of $v_{X, i}$.

LG4 Consider a sequence of vertices of the root graph $\left(v_{X, 1}, v_{X, 2}, \ldots, v_{X, n+1}\right)$, where $v_{X, 1}=v_{X, n+1}$ but all other vertices are distinct. Take the sequence of edges $\left(e_{X, 1}, e_{X, 2}, \ldots e_{X, n}\right)$ of $X$ where the edge $e_{X, i}$ connects vertices
$v_{X, i}$ and $v_{X, i+1}$. These vertices and edges form a "cycle" of the graph. As a consequence of the line-graph construction, every cycle of $X$ gives rise to a cycle of equal length (number of edges) of $L(X)$. These cycles of $L(X)$ are "chordless," meaning that no two vertices of the cycle are connected by an edge that does not belong in the cycle.

For regular root-graph lattices $X$ with $n$ vertices per unit cell, each with coordination number (degree) $d$, we have additionally the following:

LG5 Given energies $E_{X}$ of $X$, its corresponding line-graph lattice $L(X)$ has energies $E_{L(X)}=\left\{E_{X}+d-2,-2\right\}$, with one or more flat bands at -2 .

LG6 The degeneracy $D$ of the flat band at -2 is given by $D=n(d-2) / 2$.

LG7 If $X$ is nonbipartite, then the flat band(s) at -2 for $L(X)$ will be gapped from the other bands.

Finally, if $X$ (under periodic boundary conditions) can be embedded on a torus such that none of its edges cross each other, then we define the faces of $X$ to be regions bounded by edges and containing no edges or vertices. Because $X$ is on a torus, the coordination number $d$ and number of vertices $n$ per unit cell then determine the number of faces is per unit cell to be equal to the band degeneracy $D$ :

LG8 The number of faces per unit cell of $X$ is also given by $n(d-2) / 2$.

We consider line-graph lattices of nonbipartite toroidal regular root-graph lattices, with flat-band degeneracy $1<D \leqslant 4$. These lattices have $C_{2}, C_{3}$, or $C_{6}$ symmetry, and can be further split into families based on their coordination number and the number of faces per unit cell that are bounded by an even number of edges ("even-sided faces"). We find that lattices in the same family have the same representation of the associated flat bands. More specifically, these three characteristics define which graph-element type-vertex, edge, or face-is located at each maximal Wyckoff position of the root-graph lattice unit cell. Maximal Wyckoff positions in a space group are the high-symmetry points in real space with the little groups-under which they are invariant-as maximal subgroups of the space group. Each element type (vertex, edge, or face) then determines the so-called real-space invariants (RSIs) of the flat band at each maximal Wyckoff position, from which the representation and topology follow [23]. Furthermore, for $D=3$ and $D=4$ flat bands we consider various perturbations to reduce the degeneracy and identify a class of perturbations that produces fragile topological flat bands.

In discussing our framework, we will use two elucidating examples, shown in Fig. 1; additional examples are included in Appendix F of the Supplemental Material [59]. For example 1 , we take the line graph of the triangle lattice, which has coordination number 6 , zero even-sided faces, and $C_{6}$ symmetry. It also has one vertex and two faces per unit cell; therefore, the corresponding line-graph lattice has a $D=2$-fold degeneracy of its flat bands at energy -2 . For example 2, we take the line graph of the heptagon-heptagon-pentagon-pentagon lattice with $M_{x}$ and $M_{y}$ mirror symmetries as shown in Fig. 1. This root-graph lattice has coordination number 3, zero even-sided faces, and $C_{2}$ symmetry. It also has eight vertices and four faces per unit cell; therefore, the corresponding line-graph lattice has a $D=4$-fold degeneracy of its flat bands at -2 . 

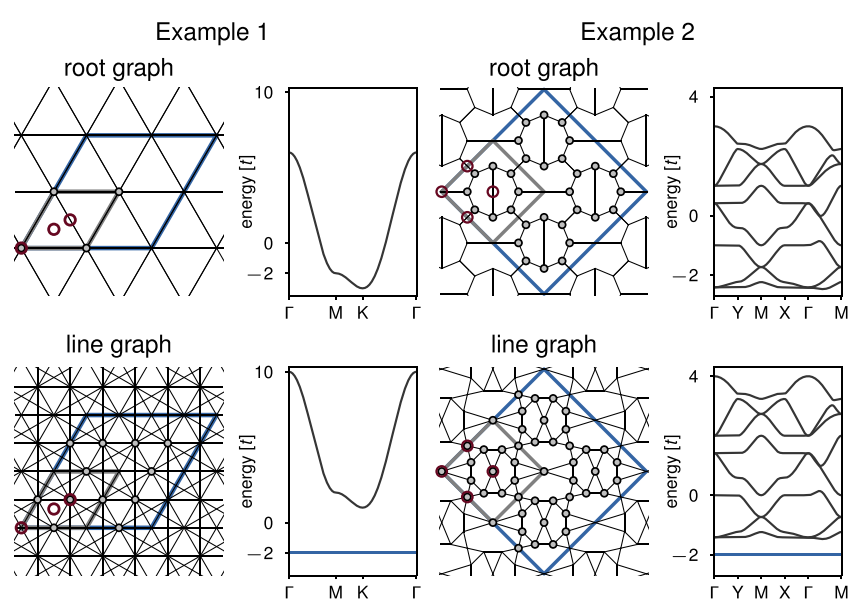

line graph
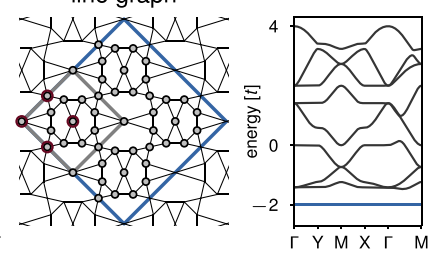

FIG. 1. The two examples of line-graph lattices described in the main text. Example 1 begins with the triangle lattice as its root graph, and example 2 begins with the heptagon-heptagon-pentagonpentagon lattice with $M_{x}$ and $M_{y}$ mirror symmetries. Upon taking the line graph of these root-graph lattices, the band spectra shift upward in energy (by $d-2$ ) and flat bands are created at -2 . Unit cells are outlined in gray, lattice vertices in a two-unit-cell by two-unit-cell region (outlined in blue) are drawn as gray circles, and the maximal Wyckoff positions of one unit cell are drawn as red circles.

\section{FROM ROOT-GRAPH LATTICE PROPERTIES TO GRAPH ELEMENT AT EACH MAXIMAL WYCKOFF POSITION}

Maximal Wyckoff positions are labeled by a number according to their multiplicity and a letter defining their position (see top row of Table I). They play a large role in the construction of EBRs. Previous works have considered which maximal

TABLE I. For the maximal Wyckoff positions associated with a given point-group symmetry, depicted in the header row, we predict how many of them have vertices (v), edges (e), or faces (f) of the root-graph lattice at these positions based on the lattice's flat-band degeneracy $D$, coordination number $d$, and number of even-sided faces per unit cell. For example, the root-graph lattice of example 2 has $C_{2}$ symmetry, $D=4$, zero even faces, and $d$ odd, so the table indicates that its four maximal Wyckoff positions should be occupied by edges; indeed, as seen in Fig. 1, this is the case. We note that for the $C_{3}$ - and $C_{6}$-symmetric lattices, we find a single lattice geometry for each cell in the table, drawn in Fig. S8 of the Supplemental Material [59]. Cells corresponding to examples 1 and 2 are in blue.

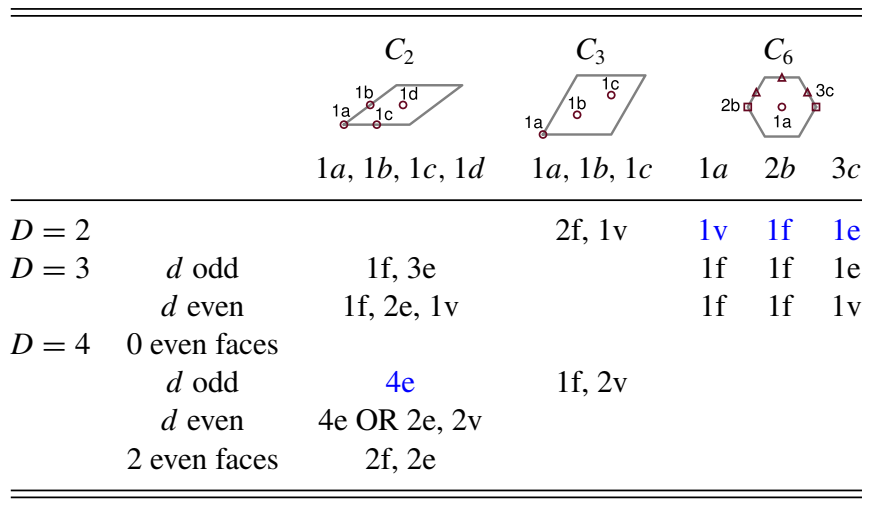

Wyckoff positions are occupied by lattice vertices (atomic orbitals) to define EBRs [1,31,32,35-37]. However, here we consider all graph elements of the lattice and whether maximal Wyckoff positions are occupied by vertices, edges, or faces of the root-graph lattice. In general, the lattices we consider contain many vertices on nonmaximal Wyckoff positions as well. As the first step in determining the properties under symmetry of the line-graph lattice flat band, we show the relationship between the root-graph lattice properties and the graph element at each maximal Wyckoff position.

The maximal Wyckoff positions for our two examples are highlighted in Fig. 1 as red circles. Example 1 has $C_{6}$ symmetry and its maximal Wyckoff positions are the $1 a, 2 b$, and $3 c$ positions, defined in Table I. In its root-graph lattice (the triangle lattice), at the $1 a$ position sits a vertex, at $2 b$ is a face, and at $3 c$ is an edge. As for example 2, its maximal Wyckoff positions are the $1 a, 1 b, 1 c$, and $1 d$ positions (Table I) resulting from its $C_{2}$ symmetry. In its root-graph lattice, at all four are edges.

More generally, we find a relationship between how many of each graph-element type are at a root-graph lattice's maximal Wyckoff positions, and the lattice's coordination number, number of even-sided faces, and symmetry. These correspondences are listed in Table I, with cells pertaining to examples 1 and 2 colored in blue. Several patterns emerge across these root-graph lattices, stated and proved in Appendix $\mathrm{C}$ of the Supplemental Material [59].

From the line-graph construction and properties LG2, LG3, and LG4 of line graphs, we can determine which graph element of the line-graph lattice is on each of maximal Wyckoff positions, given which root-graph graph element is on each maximal Wyckoff position in the root graph. For example, as seen in Fig. 1, the triangle lattice's $1 a$ maximal Wyckoff position is occupied by a vertex; $2 b$ is occupied by a triangular face, which is bounded by a cycle of length 3 ; and $3 c$ is occupied by an edge. Upon taking the line graph (see Appendix A of the Supplemental Material for details [59]), the root-graph vertex at $1 a$ gives rise to a complete subgraph at $1 a$ in the line graph, of six vertices that are pairwise fully connected by $\left(\begin{array}{l}6 \\ 2\end{array}\right)=15$ edges (property LG3 of line graphs). Similarly, the root-graph triangular face at $2 b$ gives rise to a triangular face at $2 b$ in the line graph (property LG4), and the root-graph edge at $3 c$ gives rise to a vertex at $3 c$ in the line graph (by definition of the line-graph construction).

\section{FROM MAXIMAL WYCKOFF POSITION LOCATION TYPE TO REAL-SPACE INVARIANT}

Real-space invariants (RSIs) are quantum numbers assigned to maximal Wyckoff positions and can be used to determine band topology. RSIs compute the local representation of an orbital at a Wyckoff position, which induces a set of bands in the Brillouin zone [23]. For a maximal Wyckoff position with point symmetry $C_{s}$, these eigenstates can have (single group) eigenvalues $e^{i 2 \pi k / s}$ for integer $k \in$ $[0,1, \ldots s-1]$. Here we consider RSIs for two-dimensional point-group symmetries without spin-orbit coupling and with time-reversal symmetry (TRS). Due to TRS, there is a oneto-one correspondence between eigenstates with eigenvalue $e^{ \pm i 2 \pi k / s}$, and hence we only consider $k \leqslant\lfloor s / 2\rfloor$. The RSIs 
at maximal Wyckoff position $w$ are then equal to the difference in multiplicities $m_{w, k \neq 0}^{s}$ and $m_{w, k=0}^{s}$ of these eigenstates: $\delta_{w, k^{\prime}}^{s} \equiv m_{w, k^{\prime}}^{s}-m_{w, 0}^{s}$ for $k^{\prime} \in[1, s / 2]$. We note that these RSIs are can also be written using the point group irreducible representation (orbital) notation from the Bilbao Crystallographic Server [35], but avoid this notation here for simplicity.

A real-space approach to determine the RSIs of a $C_{s}$ center is to consider local energy eigenfunctions $|\phi\rangle$ plus each of their $C_{s}$ images with a relative phase:

$$
\left|\phi_{k}\right\rangle \equiv|\phi\rangle+e^{i 2 \pi k / s} C_{s}|\phi\rangle+\cdots+\left(e^{i 2 \pi k / s} C_{s}\right)^{s-1}|\phi\rangle .
$$

Notice that each value of $k \in[0, s / 2]$ generates an eigenfunction of eigenvalue $e^{i 2 \pi k / s}$. However, some of these constructions may yield $\left|\phi_{k}\right\rangle \propto|\phi\rangle$ (with an overall phase), which occurs when $|\phi\rangle$ is a $C_{s}$ eigenstate, or vanish identically. If either of these is the case, then one or more of the RSIs will nonzero valued. To evaluate the RSIs for our line graphs, we choose a real-space flat-band eigenbasis containing so-called "cycle" and "chain" compact localized states (CLSes), which are defined in Appendix B of the Supplemental Material [59].

Figure 2 depicts the RSIs and associated CLS eigenstates at each maximal Wyckoff position for our two examples. For example 1 , we define a flat-band eigenstate $|\phi\rangle$ with nonzero amplitude on four vertices in the line-graph lattice, enclosing an even cycle around two of the triangle faces. At the $1 a$ position, we consider the sum of $|\phi\rangle$ with each of its $C_{6}$ images with a relative phase $e^{i 2 \pi k / 6}$ [see Fig. 2(a)]. Of the integers $k \in[0,3]$, all yield nonzero functions except for $k=0$. In particular, notice that the $C_{s}$ eigenstate constructions can vanish identically for some $k$ only if each vertex (of the line-graph lattice) where $|\phi\rangle$ has nonzero amplitude, also has nonzero amplitude for at least one of the $C_{s}$ images of $|\phi\rangle$. All other local flat-band symmetry eigenstates for the line graph of the triangle lattice involve a local energy eigenfunction $\left|\phi^{\prime}\right\rangle$ that does not have this property; therefore, the constructions $\left|\phi_{k}^{\prime}\right\rangle$ will construct the same number of eigenfunctions of each eigenvalue. Then the eigenstates $\left|\phi_{k}^{\prime}\right\rangle$ do not contribute to the RSIs of the origin $1 a$, and the RSIs are $\delta_{1 a, 1}^{6}=\delta_{1 a, 2}^{6}=\delta_{1 a, 3}^{6}=$ 1 . The same procedure for the $2 b$ and $3 c$ positions yields RSIs of $\delta_{2 b, 1}^{6}=0$ and $\delta_{3 c, 1}^{6}=-1$.

In example 2, we define different local eigenstates $|\phi\rangle$ at each of the four maximal Wyckoff positions; however, each yield one more $C_{2}$ eigenstate of eigenvalue +1 than -1 . Again, all other local eigenstates of the chosen Wyckoff position create an equal number of eigenfunctions of each \pm eigenvalue, so the RSIs are $\delta_{1 a, 1}^{2}=\delta_{1 b, 1}^{2}=\delta_{1 c, 1}^{2}=\delta_{1 d, 1}^{2}=$ -1 .

These RSI values at each maximal Wyckoff position can be generalized to those in our other line-graph lattices based upon the line-graph graph element sitting on the maximal Wyckoff position and the point-group symmetry; we tabulate these relationships in Table II and prove them in Appendix C of the Supplemental Material [59].

\section{FROM RSIS TO REPRESENTATION}

Once the RSIs have been determined, it is straightforward to solve for the representation. RSIs are linear invariant

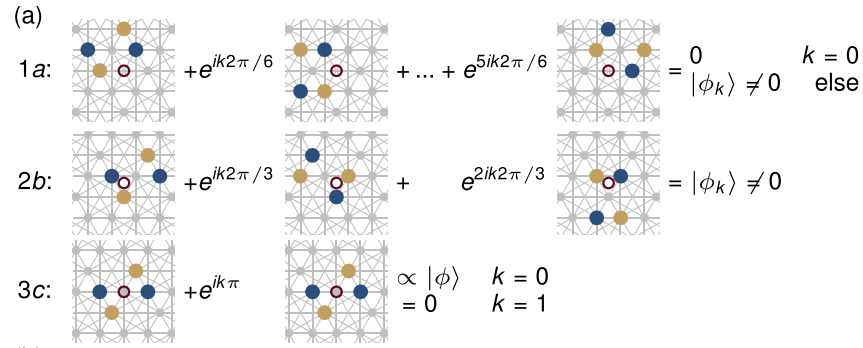

(b)

Example 1
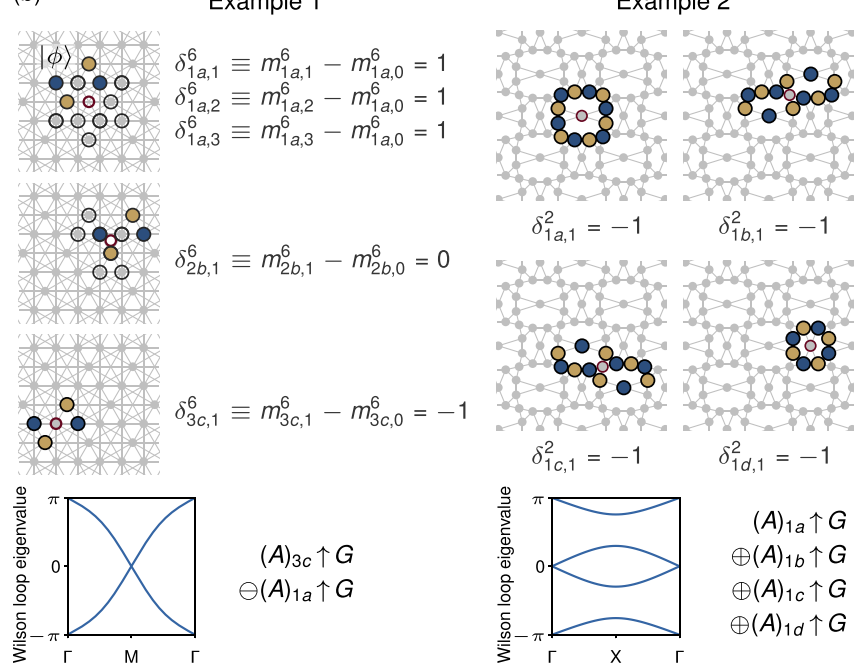

$(A)_{3 c} \uparrow G$

$\ominus(A)_{1 a} \uparrow G$

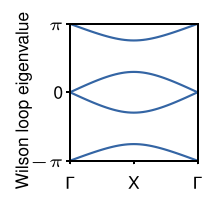

$(A)_{1 a} \uparrow G$

$\oplus(A)_{1 b} \uparrow G$

$\oplus(A)_{1 c} \uparrow G$

$\oplus(A)_{1 d} \uparrow G$

FIG. 2. The point-group-symmetric eigenstates local to each maximal Wyckoff position (red circles) depict the real-space invariants (RSIs) for each position, as demonstrated by our two examples. Here, the local flat-band eigenfunctions $|\phi\rangle$ are based on compact localized states (CLSes; see main text). They are real valued and depicted by the colored circles, with blue (yellow) circles denoting relative amplitude $+1(-1)$. (a) $C_{s}$ flat-band eigenstate construction from flat-band energy eigenstates, as described in the main text, for each maximal Wyckoff position in example 1. The flat-band energy eigenstate $|\phi\rangle$ and its $C_{s}$ images are represented graphically. (b) RSI determination for examples 1 and 2 based on the multiplicities of $C_{s}$ flat-band eigenstates of each eigenvalue. Circles outlined in black highlight vertices where at least one of the $C_{s}$ images of $|\phi\rangle$ have nonzero amplitude. The representation follows directly from these RSIs, and we find odd Wilson loop winding when the representation involves a difference of EBRs as in example 1.

under induction, so they also describe the differences in EBR multiplicities $\widetilde{m}_{w, k}$ for EBRs induced from the orbitals corresponding to $C_{s}$ eigenvalue $e^{i 2 \pi k / s}$ at maximal Wyckoff positions $w$. There is also an additional constraint on the total number of flat bands $D$,

$$
\sum_{w, k \in[0, s / 2]} m_{w, k} \tilde{m}_{w, k}=D,
$$

where $m_{w, k}$ is the dimension of the induced EBR at maximal Wyckoff position $w$. The representations for various families of line-graph lattices are derived in Appendix E of the Supplemental Material [59]; we now explicitly consider our two examples.

For $C_{6}$-symmetric lattices, we have $m_{1 a, 0}=1, m_{2 b, 0}=2$, and $m_{3 c, 0}=3$. In example 1 , with Eq. (3) we find $\widetilde{m}_{1 a, 0}=$ $-1, \widetilde{m}_{3 c, 0}=1$, and hence the representation can be written 
TABLE II. For a maximal Wyckoff position $w$ associated with a given point-group symmetry, indicated in the header row, its RSIs can be determined based on the line-graph graph element occupying $w$.

\begin{tabular}{lccc}
\hline \hline & $C_{2}$ & $C_{3}$ & $C_{6}$ \\
\hline Vertex & $\delta_{w, 1}^{2}=-1$ & $\delta_{w, 1}^{3}=0$ & $\delta_{1 a, 1}^{6}=\delta_{1 a, 2}^{6}=\delta_{1 a, 3}^{6}=0$ \\
Complete & $\delta_{w, 1}^{2}=+1$ & $\delta_{w, 1}^{3}=+1$ & $\delta_{1 a, 1}^{6}=\delta_{1 a, 2}^{6}=\delta_{1 a, 3}^{6}=+1$ \\
Subgraph & & & \\
Face & $\delta_{w, 1}^{2}=0$ & $\delta_{w, 1}^{3}=0$ & $\delta_{1 a, 1}^{6}=\delta_{1 a, 2}^{6}=\delta_{1 a, 3}^{6}=0$ \\
\hline \hline
\end{tabular}

as $(A)_{3 c} \uparrow G \ominus(A)_{1 a} \uparrow G$, where now we use the irrep notation from the Bilbao Crystallographic Server [35]. Although this decomposition is not unique, all equivalent decompositions have a negative coefficient. Because this representation can be written as a difference of EBRs, the flat bands in example 1the line graph of the triangle lattice-exhibit fragile topology. The Wilson loop for these bands exhibits winding, confirming our result [see Fig. 2(b)].

For $C_{2}$-symmetric lattices, we have $m_{1 a, 0}=m_{1 b, 0}=$ $m_{1 c, 0}=m_{1 d, 0}=1$, so in example 2 we find $\widetilde{m}_{1 a, 0}=\widetilde{m}_{1 b, 0}=$ $\widetilde{m}_{1 c, 0}=\widetilde{m}_{1 d, 0}=+1$. This yields the representation $(A)_{1 a} \uparrow$ $G \oplus(A)_{1 b} \uparrow G \oplus(A)_{1 c} \uparrow G \oplus(A)_{1 d} \uparrow G$ and we cannot conclude that these fourfold-degenerate bands of example 2 exhibit fragile topology. Correspondingly, the Wilson loop eigenvalues show no odd winding.

At this point, among our line-graph lattices we find one $D=2$ lattice with fragile topological flat bands-the line graph of the triangle lattice - and one $D=2$ lattice which admits a Wannier representation-the line graph of the nonagon-triangle lattice (see Appendix $\mathrm{F}$ of the Supplemental Material [59]). We also find that all flatband representations for the $D=3$ and $D=4$ line-graph lattices considered are a sum of EBRs, indicating that each group of bands may be topologically trivial. However, we can split the flat-band band degeneracy for these $D>2$ line-graph lattices and characterize the resulting band topology. We examine perturbations that leave twofolddegenerate gapped flat bands at the original flat-band energy -2 . We refer to this process as "splitting the bands."

\section{SPLITTING THE BANDS}

To begin, we note that on-site-energy perturbations can successfully split the bands for $D=3$ and $D=4$ into flat band(s) and dispersive bands, for example, as in the left of Fig. 3. However, the remaining flat band(s) are still EBRs or sums of EBRs. Because these perturbations are localized on single vertices, they will not change the existing Wannier representation for the flat-band eigenfunctions.

Therefore, we focus on symmetry-preserving perturbations consisting of new hoppings. For $D=4$ line-graph lattices with $C_{2}$ symmetry, such as example 2 (see Fig. 3), we find that the bands can always be split into a set of two flat bands and two dispersive ones. More specifically, every $D=4$ linegraph lattice has a root-graph unit cell with either two evenand two odd-sided faces (the "2e2o" family) or four oddsided faces (the " 40 " family). For 2e2o lattices, the flat-band degeneracy can be split by introducing a hopping between

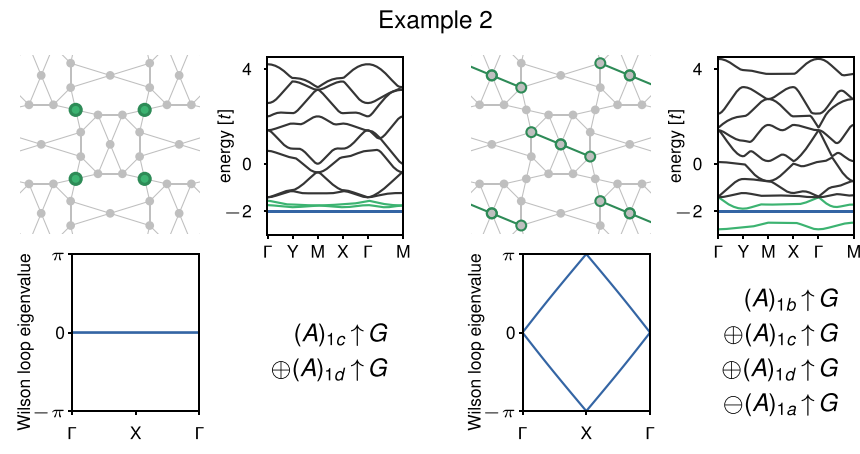

FIG. 3. By adding a perturbation (green) consisting of (left) onsite energies or (right) additional hoppings, we can split the $D=4$ degeneracy of example 2 to create twofold-degenerate gapped flat bands. We predict the topology of these bands given the perturbation; here the hopping perturbation leaves bands with fragile topology, as seen in the representation and Wilson loop winding.

the two vertices that are each adjacent to both even-sided faces, as shown in Fig. S12(a) [59]. For 4o lattices, it can be split through two hoppings that (1) are $C_{2}$ images of one another and share a vertex at a maximal Wyckoff position, (2) each extend across a single face, and (3) are between vertices adjacent to all four faces. A construction is depicted in Fig. S13(a) [59], with the result seen in the right of Fig. 3. In both families, these prescribed hoppings always exist; of course, there may also be alternate hopping perturbations for these lattices that also split the bands successfully. These claims are proved in Appendix D of the Supplemental Material [59].

By contrast, for all other line-graph lattices considered we find evidence, presented in Appendix D of the Supplemental Material [59], that the bands cannot be split into twofolddegenerate gapped flat bands. For example, in $D=3$ lattices with $C_{2}$ symmetry, it seems that hopping perturbations can at best split the three bands into one flat band, sharing a band touch with one dispersive band, and one other, separate, dispersive band.

For bands that can be split, their postperturbation representation can be predicted with the same formalism. Intuitively, a perturbation splits the bands by inducing level repulsion between identical atomic orbitals; indeed, this is the case for example 2, as seen in the right of Fig. 3, where the perturbed bands each have a representation induced from an orbital on the same maximal Wyckoff positon, 1a. Level repulsion can also occur between two orbitals on general (nonmaximal) Wyckoff positions, which is equivalent to one $s$ and one $p$ orbital for a maximal Wyckoff position $w$ of multiplicity 1 (see Appendix E of the Supplemental Material and the last two rows of Table S3 [59]). We also find that bands with fragile topology can be realized through our constructed hopping perturbations on the 4o lattices, but not on the $2 \mathrm{e} 2 \mathrm{o}$ lattices; proofs are in Appendix E of the Supplemental Material [59]. There we also tabulate representations for perturbed $D=3 C_{2}$-symmetric lattices, where if the perturbation is symmetry preserving and involves two vertices on a face that sits on a maximal Wyckoff position, then the resulting band pair exhibits fragile topology. 


\section{CONCLUSION}

We have shown how to predict the representation of the energy $=-2$ flat bands for line-graph lattices of planar regular root-graph lattices where these bands are gapped from the rest of the spectrum. These predictions only require knowledge of purely geometric qualities of the root-graph lattice structure. We further demonstrate that in cases of flat bands with fourfold flat-band degeneracy, perturbations to the line graph always exist to partially break the degeneracy and leave doubly degenerate gapped flat bands, whose representation can also be predicted. Of the line-graph lattices considered in this work, we find one $D=2$ lattice with fragile topological flat bands - the line graph of the triangle lattice - and a family of $D=4$ lattices with fragile topological flat bands after one of a class of specific perturbations - the 4o family. We also find that for our $D=3$ lattices, there exists a perturbation that yields a pair of fragile topological bands (one flat and one dispersive).

Possible extensions of this work, some of which are briefly discussed in Appendix G of the Supplemental Material [59], include extending the formalism to higher degeneracies $D>$ 4, which will also allow for the treatment of lattices with $C_{4}$ symmetry, and the addition of $p$ - and $d$-orbital hopping to the tight-binding model. Other extensions include considering irregular root-graph lattices where vertices can have differing coordination number, nontoroidal root-graph lattices where edges can cross each other without meeting at a vertex, or proving the results of alternate hopping perturbation construc- tions. Similar work has been done on the band topology of ungapped flat bands in line graph and split graphs of bipartite lattices, after the bands are gapped by introducing spin-orbit coupling [60].

Our results dictate the course of quantum simulation of fragile topology in line-graph lattices, a system particularly suitable for the platform of microwave quantum circuits. Coplanar waveguide resonators have been used to create various line-graph lattice geometries in two dimensions; in particular, the isotropic three-way capacitor is a wellestablished and straightforward circuit element to realize such lattices with $d=3[61,62]$. By creating artificial materials with these crystalline structures using microwave resonators, it may be possible to probe the physics of fragile topology in flat electronic bands.

\section{ACKNOWLEDGMENTS}

We acknowledge support from NSF-MRSEC (No. DMR1420541) and the Princeton Center for Complex Materials, ARO-MURI (No. W911NF-15-1-0397), the NationalKey R\&D Program of China (No. 2016YFA0300600), the National Natural Science Foundation of China (No. 11734003), DOE (No. DE-SC0016239), the Schmidt Fund for Innovative Research, Simons Investigator Grant (No. 404513), the Packard Foundation, NSF-EAGER (No. DMR-1643312), ONR (No. N00014-20-1-2303), the Gordon and Betty Moore Foundation (No. GBMF8685), and US-Israel BSF (No. 2018226).
[1] B. Bradlyn, L. Elcoro, J. Cano, M. G. Vergniory, Z. Wang, C. Felser, M. I. Aroyo, and B. A. Bernevig, Topological quantum chemistry, Nature (London) 547, 298 (2017).

[2] H. C. Po, H. Watanabe, and A. Vishwanath, Fragile Topology and Wannier Obstructions, Phys. Rev. Lett. 121, 126402 (2018).

[3] J. Cano, B. Bradlyn, Z. Wang, L. Elcoro, M. G. Vergniory, C. Felser, M. I. Aroyo, and B. A. Bernevig, Topology of Disconnected Elementary Band Representations, Phys. Rev. Lett. 120, 266401 (2018).

[4] B. Bradlyn, Z. Wang, J. Cano, and B. A. Bernevig, Disconnected elementary band representations, fragile topology, and Wilson loops as topological indices: An example on the triangular lattice, Phys. Rev. B 99, 045140 (2019).

[5] M. B. de Paz, M. G. Vergniory, D. Bercioux, A. García-Etxarri, and B. Bradlyn, Engineering fragile topology in photonic crystals: Topological quantum chemistry of light, Phys. Rev. Res. 1, 032005(R) (2019).

[6] Y. Hwang, J. Ahn, and B.-J. Yang, Fragile topology protected by inversion symmetry: Diagnosis, bulk-boundary correspondence, and Wilson loop, Phys. Rev. B 100, 205126 (2019).

[7] A. Bouhon, A. M. Black-Schaffer, and R.-J. Slager, Wilson loop approach to fragile topology of split elementary band representations and topological crystalline insulators with time-reversal symmetry, Phys. Rev. B 100, 195135 (2019).

[8] A. Alexandradinata, J. Höller, C. Wang, H. Cheng, and L. Lu, Crystallographic splitting theorem for band representations and fragile topological photonic crystals, Phys. Rev. B 102, 115117 (2020).

[9] Z.-D. Song, L. Elcoro, Y.-F. Xu, N. Regnault, and B. A. Bernevig, Fragile Phases as Affine Monoids: Classification and Material Examples, Phys. Rev. X 10, 031001 (2020).

[10] J. L. Mañes, Fragile phonon topology on the honeycomb lattice with time-reversal symmetry, Phys. Rev. B 102, 024307 (2020).

[11] D. V. Else, H. C. Po, and H. Watanabe, Fragile topological phases in interacting systems, Phys. Rev. B 99, 125122 (2019).

[12] S. Liu, A. Vishwanath, and E. Khalaf, Shift Insulators: Rotation-Protected Two-Dimensional Topological Crystalline Insulators, Phys. Rev. X 9, 031003 (2019).

[13] K. Latimer and C. Wang, Correlated fragile topology: A Parton approach, arXiv:2007.15605.

[14] C. L. Kane and E. J. Mele, $Z_{2}$ Topological Order and the Quantum Spin Hall Effect, Phys. Rev. Lett. 95, 146802 (2005).

[15] B. A. Bernevig, T. L. Hughes, and S.-C. Zhang, Quantum spin Hall effect and topological phase transition in $\mathrm{HgTe}$ quantum wells, Science 314, 1757 (2006).

[16] M. König, S. Wiedmann, C. Brüne, A. Roth, H. Buhmann, L. W. Molenkamp, X.-L. Qi, and S.-C. Zhang, Quantum spin Hall insulator state in HgTe quantum wells, Science 318, 766 (2007).

[17] L. Fu, C. L. Kane, and E. J. Mele, Topological Insulators in Three Dimensions, Phys. Rev. Lett. 98, 106803 (2007).

[18] H. Zhang, C.-X. Liu, X.-L. Qi, X. Dai, Z. Fang, and S.-C. Zhang, Topological insulators in $\mathrm{Bi}_{2} \mathrm{Se}_{3}, \mathrm{Bi}_{2} \mathrm{Te}_{3}$ and $\mathrm{Sb}_{2} \mathrm{Te}_{3}$ 
with a single Dirac cone on the surface, Nat. Phys. 5, 438 (2009).

[19] Y. L. Chen, J. G. Analytis, J.-H. Chu, Z. K. Liu, S.-K. Mo, X. L. Qi, H. J. Zhang, D. H. Lu, X. Dai, Z. Fang, S. C. Zhang, I. R. Fisher, Z. Hussain, and Z.-X. Shen, Experimental realization of a three-dimensional topological insulator, $\mathrm{Bi}_{2} \mathrm{Te}_{3}$, Science $\mathbf{3 2 5}$, 178 (2009).

[20] Y. Xia, D. Qian, D. Hsieh, L. Wray, A. Pal, H. Lin, A. Bansil, D. Grauer, Y. S. Hor, R. J. Cava, and M. Z. Hasan, Observation of a large-gap topological-insulator class with a single Dirac cone on the surface, Nat. Phys. 5, 398 (2009).

[21] A. Kitaev, Periodic table for topological insulators and superconductors, in Advances in Theoretical Physics: Landau Memorial Conference, AIP Conf. Proc. No. 1134 (AIP, New York, 2009), pp. 22-30.

[22] A. P. Schnyder, S. Ryu, A. Furusaki, and A. W. W. Ludwig, Classification of topological insulators and superconductors in three spatial dimensions, Phys. Rev. B 78, 195125 (2008).

[23] Z.-D. Song, L. Elcoro, and B. A. Bernevig, Twisted bulkboundary correspondence of fragile topology, Science 367, 794 (2020).

[24] V. Peri, Z.-D. Song, M. Serra-Garcia, P. Engeler, R. Queiroz, X. Huang, W. Deng, Z. Liu, B. A. Bernevig, and S. D. Huber, Experimental characterization of fragile topology in an acoustic metamaterial, Science 367, 797 (2020).

[25] F. Xie, Z. Song, B. Lian, and B. A. Bernevig, TopologyBounded Superfluid Weight in Twisted Bilayer Graphene, Phys. Rev. Lett. 124, 167002 (2020).

[26] X. Hu, T. Hyart, D. I. Pikulin, and E. Rossi, Geometric and Conventional Contribution to the Superfluid Weight in Twisted Bilayer Graphene, Phys. Rev. Lett. 123, 237002 (2019).

[27] A. Julku, T. J. Peltonen, L. Liang, T. T. Heikkilä, and P. Törmä, Superfluid weight and Berezinskii-Kosterlitz-Thouless transition temperature of twisted bilayer graphene, Phys. Rev. B 101, 060505(R) (2020).

[28] T. Hazra, N. Verma, and M. Randeria, Bounds on the Superconducting Transition Temperature: Applications to Twisted Bilayer Graphene and Cold Atoms, Phys. Rev. X 9, 031049 (2019).

[29] J. Herzog-Arbeitman, Z.-D. Song, N. Regnault, and B. A. Bernevig, Hofstadter Topology: Noncrystalline Topological Materials at High Flux, Phys. Rev. Lett. 125, 236804 (2020).

[30] B. Lian, F. Xie, and B. A. Bernevig, Landau level of fragile topology, Phys. Rev. B 102, 041402(R) (2020).

[31] H. C. Po, A. Vishwanath, and H. Watanabe, Symmetry-based indicators of band topology in the 230 space groups, Nat. Commun. 8, 50 (2017).

[32] J. Kruthoff, J. de Boer, J. van Wezel, C. L. Kane, and R.-J. Slager, Topological Classification of Crystalline Insulators Through Band Structure Combinatorics, Phys. Rev. X 7 , 041069 (2017).

[33] J. Zak, Symmetry Specification of Bands in Solids, Phys. Rev. Lett. 45, 1025 (1980).

[34] J. Zak, Band representations of space groups, Phys. Rev. B 26, 3010 (1982).

[35] L. Elcoro, B. Bradlyn, Z. Wang, M. G. Vergniory, J. Cano, C. Felser, B. A. Bernevig, D. Orobengoa, G. de la Flor, and M. I. Aroyo, Double crystallographic groups and their rep- resentations on the Bilbao Crystallographic Server, J. Appl. Crystallogr. 50, 1457 (2017).

[36] J. Cano, B. Bradlyn, Z. Wang, L. Elcoro, M. G. Vergniory, C. Felser, M. I. Aroyo, and B. A. Bernevig, Building blocks of topological quantum chemistry: Elementary band representations, Phys. Rev. B 97, 035139 (2018).

[37] M. G. Vergniory, L. Elcoro, Z. Wang, J. Cano, C. Felser, M. I. Aroyo, B. A. Bernevig, and B. Bradlyn, Graph theory data for topological quantum chemistry, Phys. Rev. E 96, 023310 (2017).

[38] T. Neupert, L. Santos, C. Chamon, and C. Mudry, Fractional Quantum Hall States at Zero Magnetic Field, Phys. Rev. Lett. 106, 236804 (2011).

[39] Y.-F. Wang, H. Yao, C.-D. Gong, and D. N. Sheng, Fractional quantum Hall effect in topological flat bands with Chern number two, Phys. Rev. B 86, 201101(R) (2012).

[40] N. Regnault and B. A. Bernevig, Fractional Chern Insulator, Phys. Rev. X 1, 021014 (2011).

[41] K. Sun, Z. Gu, H. Katsura, and S. Das Sarma, Nearly Flatbands with Nontrivial Topology, Phys. Rev. Lett. 106, 236803 (2011).

[42] R. Bistritzer and A. H. MacDonald, Moiré bands in twisted double-layer graphene, Proc. Natl. Acad. Sci. USA 108, 12233 (2011).

[43] J. Ahn, S. Park, and B.-J. Yang, Failure of Nielsen-Ninomiya Theorem and Fragile Topology in Two-Dimensional Systems with Space-Time Inversion Symmetry: Application to Twisted Bilayer Graphene at Magic Angle, Phys. Rev. X 9, 021013 (2019).

[44] H. C. Po, L. Zou, T. Senthil, and A. Vishwanath, Faithful tightbinding models and fragile topology of magic-angle bilayer graphene, Phys. Rev. B 99, 195455 (2019).

[45] J. Kang and O. Vafek, Strong Coupling Phases of Partially Filled Twisted Bilayer Graphene Narrow Bands, Phys. Rev. Lett. 122, 246401 (2019).

[46] Z. Song, Z. Wang, W. Shi, G. Li, C. Fang, and B. A. Bernevig, All Magic Angles in Twisted Bilayer Graphene are Topological, Phys. Rev. Lett. 123, 036401 (2019).

[47] J. Liu, J. Liu, and X. Dai, Pseudo Landau level representation of twisted bilayer graphene: Band topology and implications on the correlated insulating phase, Phys. Rev. B 99, 155415 (2019).

[48] D. Leykam, A. Andreanov, and S. Flach, Artificial flat band systems: From lattice models to experiments, Adv. Phys.: X 3, 1473052 (2018).

[49] N. Bultinck, E. Khalaf, S. Liu, S. Chatterjee, A. Vishwanath, and M. P. Zaletel, Ground State and Hidden Symmetry of Magic-Angle Graphene at Even Integer Filling, Phys. Rev. X 10, 031034 (2020).

[50] B. Lian, Z.-D. Song, N. Regnault, D. K. Efetov, A. Yazdani, and B. A. Bernevig, TBG IV: Exact insulator ground states and phase diagram of twisted bilayer graphene, arXiv:2009.13530.

[51] T. Zhang, Y. Jiang, Z. Song, H. Huang, Y. He, Z. Fang, H. Weng, and C. Fang, Catalogue of topological electronic materials, Nature (London) 566, 475 (2019).

[52] J. Liu, Z. Ma, J. Gao, and X. Dai, Quantum Valley Hall Effect, Orbital Magnetism, and Anomalous Hall Effect in 
Twisted Multilayer Graphene Systems, Phys. Rev. X 9, 031021 (2019).

[53] K. Hejazi, X. Chen, and L. Balents, Hybrid Wannier Chern bands in magic angle twisted bilayer graphene and the quantized anomalous Hall effect, arXiv:2007.00134.

[54] Z.-D. Song, B. Lian, N. Regnault, and B. A. Bernevig, TBG II: Stable symmetry anomaly in twisted bilayer graphene, arXiv:2009.11872.

[55] B. A. Bernevig, Z.-D. Song, N. Regnault, and B. Lian, TBG III: Interacting Hamiltonian and exact symmetries of twisted bilayer graphene, arXiv:2009.12376.

[56] E. H. Lieb, Two Theorems on the Hubbard Model, Phys. Rev. Lett. 62, 1201 (1989).

[57] D. Cvetkovic, P. Rowlinson, and S. Simic, Spectral Generalizations of Line Graphs: On Graphs with Least Eigenvalue -2 (Cambridge University Press, Cambridge, UK, 2004).
[58] I. Carusotto, A. A. Houck, A. J. Kollár, P. Roushan, D. I. Schuster, and J. Simon, Photonic materials in circuit quantum electrodynamics, Nat. Phys. 16, 268 (2020).

[59] See Supplemental Material at http://link.aps.org/supplemental/ 10.1103/PhysRevResearch.2.043414 for additional examples and proofs of claims made in the main text.

[60] D.-S. Ma, Y. Xu, C. S. Chiu, N. Regnault, A. A. Houck, Z. Song, and B. A. Bernevig, Spin-orbit-induced topological flat bands in line and split graphs of bipartite lattices, arXiv:2008.08231v2 [Phys. Rev. Lett (to be published)].

[61] D. L. Underwood, W. E. Shanks, A. C. Y. Li, L. Ateshian, J. Koch, and A. A. Houck, Imaging Photon Lattice States by Scanning Defect Microscopy, Phys. Rev. X 6, 021044 (2016).

[62] A. J. Kollár, M. Fitzpatrick, and A. A. Houck, Hyperbolic lattices in circuit quantum electrodynamics, Nature (London) 571, 45 (2019). 